\title{
CRESCIMENTO DE ESPÉCIES ARBÓREAS EM UMA FLORESTA NATURAL DE TERRA FIRME APÓS A COLHEITA DE MADEIRA E TRATAMENTOS SILVICULTURAIS, NO MUNICÍPIO DE PARAGOMINAS, PARÁ, BRASIL
}

\author{
GROWTH RATE OF TREE SPECIES IN A TERRA FIRME NATURAL FOREST AFTER LOGGING \\ AND SILVICULTURAL TREATMENTS, IN THE MUNICIPALITY OF PARAGOMINAS, PARÁ, \\ BRAZIL
}

\begin{abstract}
Deivison Venicio Souza ${ }^{1}$ João Olegário Pereira de Carvalho ${ }^{2}$ Fernanda da Silva Mendes ${ }^{3}$ Lia de Oliveira Melo ${ }^{4}$ José Natalino Macedo Silva ${ }^{5}$ Fernando Cristóvam da Silva Jardim ${ }^{5}$
\end{abstract}

\section{RESUMO}

Este trabalho teve como objetivo avaliar os efeitos de tratamentos silviculturais sobre o crescimento de uma floresta natural de terra firme explorada usando técnicas de impacto reduzido. A pesquisa foi conduzida na Área de Manejo Florestal (AMF) da Fazenda Rio Capim, pertencente à empresa Cikel Brasil Verde Madeiras Ltda., localizada no município de Paragominas, Estado do Pará. A área experimental correspondeu a 500 ha, nos quais foram estabelecidos cinco tratamentos (100 ha cada) com quatro repetições ( 25 ha cada) seguindo um delineamento completamente ao acaso. As repetições foram distribuídas aleatoriamente na amostra de 500 ha na AMF. Foram avaliados os seguintes tratamentos: T1 - exploração de impacto reduzido (EIR) + desbaste de liberação clássico e corte de cipós; T2 - EIR + desbaste de liberação modificado e corte de cipós; T3 - EIR + corte de cipós; T6 - apenas EIR; T7 - floresta não explorada (testemunha). Nos quatro anos de monitoramento da floresta, após a colheita de madeira e aplicação dos tratamentos silviculturais, T2 e T3 tiveram as maiores taxas de crescimento em diâmetro. Contudo, o período de quatro anos não é suficiente para indicar o tratamento "mais adequado", com base no crescimento em diâmetro, em resposta à anelagem de árvores e corte de cipós. Em nível de espécie, o crescimento variou entre e dentro dos tratamentos experimentais. Em geral, as menores taxas de crescimento em diâmetro ocorreram na floresta não explorada (T7).

Palavras-chave: incremento diamétrico; silvicultura pós-colheita; anelagem de árvores; corte de cipós.

\begin{abstract}
This paper deals with the effects of silvicultural treatments on growth rates of a terra firme natural forest after reduced impact logging (RIL). The study was carried out in the Rio Capim Forest Management Unit, which belongs to Cikel Brasil Verde Madeiras Ltda., located in the municipality of Paragominas, PA. The experimental area comprised 500 ha, where five treatments (100 ha each) each with four replications (25 ha each) were established. The replications were randomly distributed in the 500 ha sample area. The following treatments were applied: T1 - RIL + classical liberation thinning (girdling of competing trees) and climber cutting; T2 - RIL + modified liberation thinning (girdling of competing trees) and climber

1 Engenheiro Florestal, MSc., Professor da Faculdade de Engenharia Florestal, Universidade Federal do Pará, Rua Coronel José Porfírio, 2105, CEP 68370-000, Altamira (PA), Brasil. deivisonvs@ufpa.br

2 Engenheiro Florestal, Dr., Universidade Federal Rural da Amazônia, Estrada do 40 Horas, 135, Villa Firenze 0718, CEP 67120-370, Ananindeua (PA), Brasil. olegario@pq.cnpq.br

3 Engenheira Florestal, MSc., Doutoranda em Engenharia Florestal, Professora do Departamento de Tecnologia e Recursos Naturais, Universidade do Estado do Pará, Travessa Enéas Pinheiro, 2626, CEP 66095-100, Belém (PA), Brasil.mendes.fsm@gmail.com

4 Engenheira Florestal, $\mathrm{Dr}^{\mathrm{a}}$, Universidade Federal do Oeste do Pará, Rua Vera Paz s/n, CEP 68035-110, Santarém, (PA), Brasil.lcolivei@gmail.com

5 Engenheiro Florestal, Dr., Universidade Federal Rural da Amazônia, Av. Tancredo Neves, 2501, CEP 66077-830, Belém (PA), Brasil. silvanatalino734@gmail.com/ fernando.jardim@ufra.edu.br
\end{abstract}

Recebido para publicação em 2/12/2011 e aceito em 9/12/2013 
cutting; T3 - RIL + climber cutting; T6 - only RIL; T7 - unlogged forest (control). In the four years of monitoring forest, after logging and silvicultural treatments, T2 and T3 had the highest diameter growth rates. But the four-year period is not enough to suggest the best treatment, based on diameter growth rate, in response to girdling trees and climber cutting. At species level, growth rate varied between treatments and within treatments. In general, the lower diameter growth rates were observed in the unlogged forest. Keywords: diameter increment; post-harvesting silviculture; tree girdling; climber cutting.

\section{INTRODUÇÃO}

Nos últimos anos, as florestas tropicais têm sido alvo de muita preocupação, do ponto de vista ambiental, principalmente pela velocidade com que esses biomas estão sendo modificados ou destruídos pela ação antrópica. Nesse contexto, a promoção do manejo florestal baseado em princípios de rendimento sustentável, o qual se baseia na capacidade do povoamento florestal produzir, ao longo do tempo, um estoque compatível ao explorado, constitui-se no desafio atual e futuro da pesquisa para a conservação dos recursos florestais.

Em termos silviculturais, o grande desafio do manejo de florestas tropicais é ajustar um modelo silvicultural que garanta suficiente regeneração natural e aumento das taxas de crescimento de espécies arbóreas desejáveis, atendo-se, entre outros aspectos, à profusão exagerada da regeneração de espécies indesejáveis e/ou invasoras que possam afetar a sustentabilidade da produção madeireira.

As atividades silviculturais pós-colheita são praticamente inexistentes na Amazônia brasileira. Estudos conduzidos na região sobre a aplicação de tratamentos silviculturais focalizam suas implicações ecológicas (JARDIM et al., 1996; OLIVEIRA et al., 2005; ALVINO et al., 2006), a eficácia das técnicas silviculturais aplicadas (COSTA et al., 2001; SANDEL e CARVALHO, 2000; OLIVEIRA et al., 2009), e questões econômicas (COSTA et al., 2001).

Do ponto de vista silvicultural, há consenso entre pesquisadores de que os tratamentos silviculturais aumentam o crescimento e a produção de árvores em florestas tropicais (AMARAL et al., 1998; SILVA, 2001; AZEVEDO et al., 2008) e favorecem o estabelecimento da regeneração natural e o crescimento de mudas e varas que substituirão as perdas ocorridas durante a exploração (SILVA et al., 1999). No entanto, tais práticas em florestas naturais têm recebido pouca atenção da maioria dos usuários das florestas (SABOGAL et al., 2006).
A utilização de tratamentos silviculturais, com intuito de aumentar a produtividade da floresta pode representar uma ferramenta silvicultural de grande relevância para a sustentabilidade da produção florestal. Contudo, as informações acerca do comportamento das espécies florestais ainda são escassas, havendo necessidade de muitos estudos para subsidiar a prescrição de tratamentos silviculturais adequados.

No presente estudo é apresentada uma análise do incremento diamétrico de espécies arbóreas em uma floresta de terra firme da Amazônia, na qual foi realizada uma exploração de impacto reduzido e aplicados tratamentos silviculturais. $\mathrm{O}$ objetivo foi avaliar o efeito da exploração e dos tratamentos silviculturais sobre o crescimento de árvores selecionadas para as próximas colheitas, aumentando, assim, o conhecimento sobre o comportamento das espécies, bem como das florestas, submetidas às práticas silviculturais após a colheita da madeira.

\section{MATERIAIS E MÉTODO}

\section{Características da área de estudo}

O estudo foi conduzido na Fazenda Rio Capim, na Área de Manejo Florestal (AMF) pertencente à empresa Cikel Brasil Verde Madeiras Ltda., localizada no município de Paragominas, Pará, entre as coordenadas geográficas $03^{\circ} 30^{\prime} \mathrm{e}$ $03^{\circ} 45^{\prime}$ de latitude Sul e $48^{\circ} 30^{\prime}$ e $48^{\circ} 45^{\prime}$ de longitude Oeste (MACIEL et al., 2009).

O clima predominante na região é do tipo "Aw", conforme a classificação de Köppen, isto é, tropical chuvoso com estação seca bem definida, caracterizado por temperatura média anual de $27,2^{\circ} \mathrm{C}$, com umidade relativa do ar de $81 \%$ e precipitação pluviométrica com média de $1766 \mathrm{~mm}$ ano $^{-1}$, com ocorrência de menos disponibilidade hídrica no período de julho a outubro (WATRIN e ROCHA, 1992). 
Os principais solos ocorrentes no município de Paragominas são: Latossolos Amarelos; Argissolos Amarelos; Plintossolos; Gleissolos e Neossolos (RODRIGUES et al., 2003). De acordo com Veloso et al. (1991), a tipologia na área de estudo é denominada de floresta ombrófila densa (floresta equatorial úmida de terra firme).

\section{Delineamento experimental}

A área experimental na qual está inserido este estudo é constituída de sete tratamentos distribuídos em 6 UTs (Unidades de Trabalho) na UPA 07 (Unidade Produção Anual) e 8 UTs na UPA 08, da Área de Manejo Florestal da Fazenda Rio Capim, perfazendo uma amostra total de 700 ha, dos quais 600 ha foram explorados (exploração de impacto reduzido - EIR). Contudo, no presente estudo foram avaliadas as taxas de crescimento das árvores apenas em cinco tratamentos (T1, T2, T3, T6 e T7), totalizando 500 hectares. O delineamento foi inteiramente ao acaso. Cada tratamento teve quatro repetições. Cada UT foi dividida em quadrantes (quatro parcelas quadradas de $25 \mathrm{ha}$ ), que constituíram as repetições dos tratamentos, nas quais foram realizados os tratamentos silviculturais. Cada repetição de 25 ha tem uma bordadura de 4,75 ha (25 m para cada lado). A área efetiva, no centro da repetição, tem, portanto, 20,25 ha (450 m x 450 $\mathrm{m})$. Em suma, cada tratamento consistiu em: T1 - EIR + Desbaste de liberação clássico (sensu WADSWORTH, 2000) por anelagem, e corte de cipós nas árvores potenciais para futura colheita, que são árvores das espécies cuja madeira é atualmente comercializada. Este foi o tratamento mais rigoroso, pois, além da anelagem nas árvores competidoras que estavam sobrepondo-se às copas das árvores selecionadas, foram aneladas também aquelas que estavam a menos de $2 \mathrm{~m}$ de distância da árvore selecionada, assim como foi utilizada uma tabela de distância em função dos diâmetros das árvores selecionadas e de suas competidoras, para decidir se estas últimas teriam que ser aneladas;

T2 - EIR + Desbaste de liberação modificado (uma adaptação do clássico), por anelagem e corte de cipós nas árvores potenciais para futura colheita, as quais similarmente ao T1, também eram árvores das espécies cuja madeira é atualmente comercializada. Chamou-se Modificado porque não houve a anelagem das árvores que estavam a $2 \mathrm{~m}$ de distância da beneficiada, nem se utilizou a tabela de distância em função dos diâmetros;
T3 - EIR + Corte de cipós das árvores potenciais para futura colheita, que neste tratamento eram árvores de qualquer espécie, independente de sua madeira ser atualmente comercializada ou não; T4 - EIR + Plantio em clareiras, conservação de algumas mudas de regeneração natural de espécies de valor comercial existentes nas clareiras, e corte de cipós nas árvores potenciais para futura colheita; T5 - Constituído pelas atividades do T2, mais as atividades do T4;

T6 - Apenas EIR. Neste tratamento houve apenas a colheita das árvores de espécies comerciais de acordo com o Plano de Manejo da empresa.

T7 - Floresta não explorada (testemunha).

O diâmetro mínimo utilizado para selecionar as árvores para serem beneficiadas nos tratamentos silviculturais para as próximas colheitas foi de $35 \mathrm{~cm}$. As árvores, para serem beneficiadas, deveriam estar sadias e com boa forma.

A anelagem aplicada aos tratamentos foi do tipo anelagem completa que, segundo Sandel e Carvalho (2000), consiste em retirar a casca e o câmbio da árvore, com machadinha, formando um anel completo de, aproximadamente, $30 \mathrm{~cm}$ de largura, na altura de aproximadamente $1 \mathrm{~m}$ do solo. Essa atividade foi feita com terçado e/ou machadinha.

\section{Coleta de dados}

Os dados foram coletados em quatro ocasiões, nos anos de 2005, 2006, 2007 e 2009. Em todas as árvores selecionadas (DAP maior ou igual a $35 \mathrm{~cm}$ ), mediu-se o diâmetro a 1,30 $\mathrm{m}$ do solo (DAP), a forma e iluminação da copa, a presença e efeito de cipós nas árvores, de acordo com Silva et al. (2005).

\section{Análise de dados}

Como já foi mencionado, neste estudo foram avaliados os dados de apenas cinco tratamentos (T1, T2, T3, T6 e T7). O parâmetro utilizado na determinação do crescimento diamétrico das árvores selecionadas foi o IPA ${ }_{\mathrm{DAP}}$ (incremento periódico anual em DAP), calculado por meio da fórmula $\left(\mathrm{DAP}_{\text {final }}-\mathrm{DAP}_{\text {inicial }}\right) / \mathrm{t}$; em que: $\mathrm{DAP}_{\text {final }}$ : diâmetro a $1,30 \mathrm{~m}$ do solo obtido no final do intervalo de medição; $\mathrm{DAP}_{\text {inicial: }}$ : diâmetro a 1,30 $\mathrm{m}$ do solo obtido no início do intervalo de medição; t: intervalo de tempo, em anos, entre duas medições. Foram estabelecidos quatro períodos de avaliação: I 
(2005 a 2006); II (2006 a 2007); III (2007 a 2009); e IV (2005 a 2009).

Os testes de hipóteses de KolmogorovSmirnov e Levene foram realizados para avaliar as suposições de normalidade e homocedasticidade de variâncias, respectivamente, exigidas pelos testes paramétricos. Devido às suposições da Análise de Variância (ANOVA) não serem atendidas, foi utilizado o teste não paramétrico de Kruskal-Wallis (teste $\mathrm{H}$ ), seguido do pós-teste de comparações múltiplas de Dunn com fins de comparar as taxas de crescimento diamétrico entre os tratamentos.

\section{RESULTADOS E DISCUSSÃO}

$\mathrm{O}$ incremento médio anual das árvores tratadas e testemunhas com DAP maior ou igual a $35 \mathrm{~cm}$, nos cinco tratamentos experimentais, para os quatro períodos avaliados é apresentado na Tabela 1. De modo geral, o teste de Kruskal-Wallis revelou haver diferenças altamente significativas entre os tratamentos experimentais em todos os períodos de avaliação.

A análise do incremento periódico anual em DAP $\left(\right.$ IPA $\left._{\mathrm{DAP}}\right)$ dentro de cada tratamento experimental reporta elevada variabilidade nas taxas de crescimento diamétrico das árvores. No tratamento T7 (floresta não explorada - testemunha), por exemplo, verificam-se, nos períodos de 20052006, 2006-2007 e 2007-2009, variações nas taxas de crescimento que atingem mais de $100 \%$ do valor da média.

Essa variação é considerada normal, uma vez que as taxas de crescimento em florestas tropicais variam amplamente entre espécies, porém, é possível encontrar um alto grau de variação entre indivíduos de uma mesma espécie (SILVA et al., 1995, 1996; CARVALHO, 1997; SCHULZE et al., 2008), devido às diferenças no tamanho e no grau de iluminação das copas, além da influência de fatores genéticos (CARVALHO, 1997). Além disso, a idade pode representar importante fonte de variação no crescimento das árvores, uma vez que a curva de crescimento das mesmas é sigmoide e não linear, com taxas de crescimento que variam na medida em que o tempo transcorre.

Schulze et al. (2008) comentam que o espectro de características de cada espécie da polinização à planta adulta, passando pela semente, muda e planta jovem - é muito variado.

TABELA 1: Incremento periódico anual em DAP $\left(\mathrm{cm} \mathrm{ano}^{-1}\right)$ das árvores tratadas e testemunhas com DAP maior ou igual a $35 \mathrm{~cm}$, em 500 ha de uma floresta natural de terra firme, no município de Paragominas, Pará.

TABLE 1: Periodic annual DBH increment $\left(\mathrm{cm}\right.$ year $\left.{ }^{-1}\right)$ of the assisted trees with DBH greater than or equal to $35 \mathrm{~cm}$ in a 500 ha area of natural terra firme forest, in the municipality of Paragominas, Pará.

\begin{tabular}{ccccccccccc}
\hline \multirow{2}{*}{ Tratamentos } & $\mathrm{n}$ & \multicolumn{2}{c}{$2005-2006$} & \multicolumn{2}{c}{$2006-2007$} & \multicolumn{2}{c}{$2007-2009$} & \multicolumn{2}{c}{$2005-2009$} \\
\cline { 3 - 10 } & & $\mathrm{IPA}_{\mathrm{DAP}}$ & $\mathrm{CV}$ & $\mathrm{IPA}_{\mathrm{DAP}}$ & $\mathrm{CV}$ & $\mathrm{IPA}_{\mathrm{DAP}}$ & $\mathrm{CV}$ & IPA $_{\text {DAP }}$ & $\mathrm{CV}$ \\
\hline $\mathrm{T} 1$ & 502 & $0,44 \mathrm{ab}$ & 95,23 & $0,37 \mathrm{ab}$ & 89,88 & $0,49 \mathrm{a}$ & 74,79 & $0,42 \mathrm{ab}$ & 68,43 \\
$\mathrm{~T} 2$ & 412 & $0,48 \mathrm{~b}$ & 84,27 & $0,40 \mathrm{a}$ & 93,54 & $0,52 \mathrm{a}$ & 72,46 & $0,46 \mathrm{~b}$ & 69,01 \\
$\mathrm{~T} 3$ & 739 & $0,48 \mathrm{~b}$ & 85,81 & $0,38 \mathrm{a}$ & 93,07 & $0,52 \mathrm{a}$ & 77,12 & $0,45 \mathrm{~b}$ & 67,15 \\
$\mathrm{~T} 6$ & 739 & $0,43 \mathrm{~b}$ & 90,27 & $0,33 \mathrm{~b}$ & 98,58 & $0,42 \mathrm{~b}$ & 90,90 & $0,38 \mathrm{a}$ & 74,55 \\
$\mathrm{~T} 7$ & 1117 & $0,41 \mathrm{a}$ & 107,22 & $0,33 \mathrm{~b}$ & 100,74 & $0,31 \mathrm{c}$ & 108,72 & $0,33 \mathrm{c}$ & 81,74 \\
\hline $\mathrm{H}$ & - & \multicolumn{2}{c}{25,981} & \multicolumn{2}{c}{20,602} & 218,721 & \multicolumn{2}{c}{113,202} \\
\hline P-value & - & \multicolumn{2}{c}{0,000} & 0,000 & 0,000 & \multicolumn{2}{c}{0,000} \\
\hline
\end{tabular}

Em que: T1 = Exploração de Impacto Reduzido (EIR) + desbaste de liberação clássico e corte de cipós; T2 = EIR + desbaste de liberação modificado e corte de cipós; T3 = EIR + corte de cipós; T6 = apenas EIR; T7 = floresta não explorada (testemunha). $\mathrm{n}=$ número de indivíduos. $\mathrm{CV}=$ coeficiente de variação. $\mathrm{H}=$ valor da estatística de KruskalWallis. $P$-value $=$ indica o nível de significância do teste de Kruskal-Wallis para cada período de avaliação $(\alpha=0,05)$. Tratamentos com letras diferentes, em cada período de avaliação, são significativamente diferentes de acordo com o pós-teste de comparações múltiplas de Dunn $(\alpha=0,05)$. 
Assim, os recursos necessários à sobrevivência e ao crescimento da planta irão variar conforme as diferentes fases de ciclo de vida. Ao longo de sua vida, uma determinada árvore exigirá diferentes quantidades de luz e de outros recursos. É possível que as exigências de duas espécies sejam nitidamente diferentes em uma determinada fase de vida, mas não na fase subsequente.

No período de 2005 a 2006, foram verificadas taxas similares de incremento periódico anual em DAP (IPA ${ }_{\text {DAP }}$ ) para os 4 tratamentos nos quais ocorreram intervenções na floresta (T1, T2, T3 e T6). Diferenças significativas nas taxas de crescimento de árvores foram observadas apenas entre a testemunha não explorada (T7) em relação aos tratamentos T2 (desbaste de liberação modificado e corte de cipós), T3 (corte de cipós) e T6 (floresta apenas explorada).

As taxas de incremento verificadas para T1, T2, T3 e T6, no período de 2005 a 2006, foram mais influenciadas pela abertura do dossel promovida pela colheita de madeira. Contudo, estatisticamente, há evidência de que a redução de cipós nas árvores selecionadas também contribuiu para o aumento das taxas de IPA ${ }_{\text {DAP }}$. Por outro lado, supõe-se que os efeitos benéficos dos desbastes de liberação efetuados nos tratamentos T1 e T2 - no que diz respeito à diminuição da competição por luz, espaço e nutrientes - foram sutis, ou mesmo, ainda, insignificantes e, portanto, tiveram pouca influência sobre o crescimento das árvores no período de tempo observado.

No período 2006-2007, observaramse decréscimos nas taxas de IPA $_{\text {DAP }}$ em todos os tratamentos avaliados quando comparadas ao período antecedente (2005-2006). A redução nas taxas de crescimento verificada nos tratamentos $\mathrm{T} 1$, T2, T3 e T6, no período de 2006-2007 (três anos após a exploração florestal), pode ser evidência da diminuição dos efeitos benéficos da abertura do dossel, promovidos pela exploração florestal madeireira e, ainda, por outros fatores naturais como menor precipitação e insolação neste período, uma vez que a redução no crescimento diamétrico afetou também a testemunha (floresta não explorada). Contudo, os tratamentos T2 e T3, ainda sim, apresentaram taxas de crescimento superiores às dos T6 e T7. A taxa de crescimento em diâmetro observada no T6 diminuiu ao ponto de igualar-se à da floresta não explorada (T7).

$\mathrm{O}$ efeito benéfico da abertura do dossel florestal no crescimento de árvores foi observado em outros estudos (SILVA et al., 1995; SILVA et al., 1996; DE GRAAF et al., 1999; SILVA et al., 2001; CARVALHO et al., 2004; JARDIM e SOARES, 2010). Entretanto, similarmente ao verificado neste estudo, esses autores reportaram que os efeitos benéficos da colheita madeireira tenderam a desaparecer com o tempo. Silva et al. (1995), em estudo realizado na Floresta Nacional do Tapajós (Km 67), observaram que tais efeitos perduraram somente até três anos após a exploração florestal.

No período 2007-2009 as taxas de IPA nos tratamentos T1, T2, T3 e T6 tiveram acréscimos quando comparadas ao período antecedente. De modo similar ao período anterior (2006-2007), a avaliação do incremento em diâmetro revelou que os tratamentos combinados (colheita de madeira + corte de cipós e/ou desbastes) - T1, T2 e T3 tiveram respostas significativamente melhores quando comparados aos tratamentos T6 e T7.

Neste período (2007-2009) os efeitos benéficos dos desbastes de liberação, aplicados nos tratamentos $\mathrm{T} 1$ e $\mathrm{T} 2$, provavelmente contribuíram de forma mais expressiva do que no período antecedente (2006-2007) para o aumento das taxas

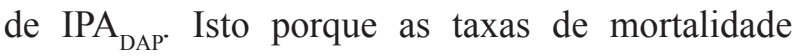
acumuladas das árvores aneladas, verificadas para ambos, no início do período de avaliação (2007), já superavam a $60 \%$ (Figura 1). Os efeitos dos desbastes de liberação sobre o crescimento diamétrico foram mais perceptíveis no decorrer do tempo, isto é, na medida em que as árvores aneladas foram perdendo a vitalidade.

O crescimento diamétrico para todo o período de acompanhamento (2005-2009) foi semelhante ao do período de 2007-2009, ou seja, os tratamentos combinados - T1, T2 e T3 - tiveram IPA $_{\text {DAP }}$ significativamente melhores do que o tratamento T7 (testemunha não explorada). Porém, o tratamento T1 não diferenciou significativamente do T6 (somente exploração de madeira).

Nos tratamentos T2 e T3, o efeito positivo no crescimento diamétrico das árvores foi observado em todos os períodos de avaliação, especialmente, quando comparados à floresta não explorada (T7).

A intervenção silvicultural pósexploratória mais efetiva entre as aplicadas no presente trabalho foi o corte de cipós, uma vez que, um ano após a sua realização, constatou-se um elevado número de árvores com cipós cortados e mortos. Logo após a realização do corte de cipós, aproximadamente $67 \%, 71 \%$ e $65 \%$ das 


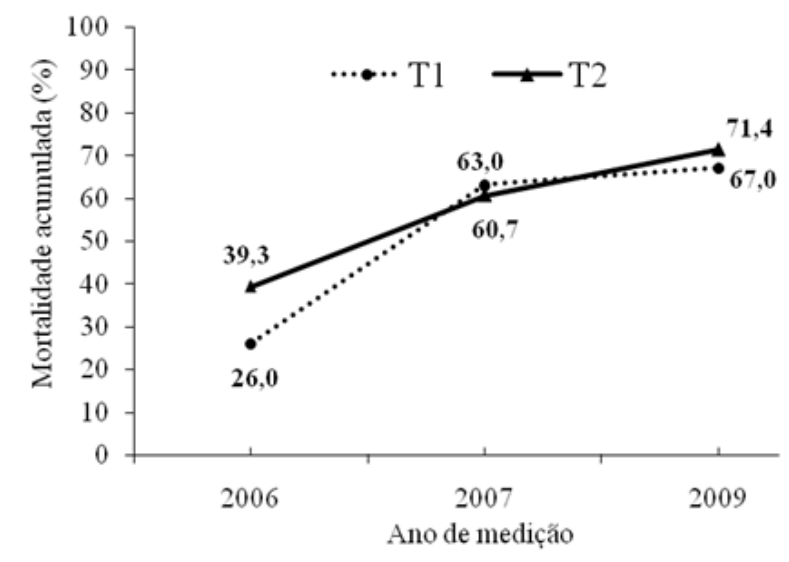

FIGURA 1: Mortalidade acumulada das árvores aneladas (DAP maior ou igual a $10 \mathrm{~cm}$ ), em 200 ha de uma floresta natural de terra firme, município de Paragominas, Pará. T1 = Exploração de Impacto Reduzido (EIR) + desbaste de liberação clássico e corte de cipós; $\mathrm{T} 2=$ EIR + desbaste de liberação modificado e corte de cipós.

FIGURE 1: Cumulative mortality of the girdled trees (DBH greater than or equal to 10 $\mathrm{cm})$ in a 200 ha of natural terra firme forest, municipality of Paragominas, Pará. $\mathrm{T} 1$ = reduced impact logging (RIL) + classical liberation thinning (girdling of competing trees) and climber cutting; T2 $=$ RIL + modified liberation thinning (girdling of competing trees) and climber cutting.

árvores liberadas nos tratamentos T1, T2 e T3, respectivamente, já se encontravam na categoria de árvores com cipós cortados e mortos (Tabela 2).

Pérez-Salicrup et al. (2001), avaliando a operação de corte de cipós em uma floresta tropical na Bolívia, verificaram que $78 \%$ das árvores encontravam-se livres de lianas em suas copas, após dois anos da aplicação do tratamento silvicultural. Os resultados deste estudo corroboram os encontrados por Pérez-Salicrup et al. (2001), visto que, decorridos dois anos notou-se que $65,74 \%$ (T1), $80,10 \%$ (T2) e 74,42\% (T3) das árvores estavam sem cipós ou com cipós cortados e mortos.

Estudos realizados em florestas tropicais têm revelado que tratamentos silviculturais aplicados após a exploração florestal podem aumentar significativamente o crescimento de árvores (WADSWORTH e ZWEEDE, 2006; PEÑACLAROS et al., 2008; VILLEGAS et al., 2009).

Peña Claros et al. (2008), em estudo realizado em floresta tropical da Bolívia, avaliaram os efeitos da aplicação de tratamentos silviculturais nas taxas de crescimento de árvores após a execução da Exploração de Impacto Reduzido EIR e constataram que as taxas de crescimento das árvores aumentaram de 9 a $27 \%$ quando praticada a silvicultura intensiva em comparação aos indivíduos submetidos somente a EIR. No presente estudo, quatro anos após a aplicação dos tratamentos silviculturais pós-exploratórios, constatou-se que as taxas de crescimento dos tratamentos com atividades combinadas foram superiores $(10,53$ a $21,05 \%$ ) às observadas no tratamento T6 (floresta apenas explorada).

Hutchinson e Wadsworth (2006), avaliando os efeitos da liberação de árvores potenciais para futura colheita em uma floresta secundária de 40 anos, na Costa Rica, constataram que as árvores liberadas cresceram cerca de um terço mais do que as testemunhas, decorridos sete anos da aplicação dos tratamentos silviculturais, o que implicaria na redução de um terço no tempo de espera para a próxima exploração florestal.

De Graaf et al. (1999) constataram que os efeitos benéficos dos tratamentos silviculturais aplicados no sistema CELOS são limitados a um período de 10 anos e, portanto, sugerem a aplicação de tratamentos silviculturais periódicos para manter as condições ótimas de crescimento das espécies potenciais para futura colheita e, ainda, conforme De Graaf (1986), induzir a regeneração natural.

Em nível de espécie, os efeitos das intervenções silviculturais (colheita de madeira e/ou corte de cipós e/ou desbastes) nas taxas de IPA $_{\text {DAP }}$ mostraram-se não significativos para as espécies Protium subserratum e Laetia procera, isto porque seus incrementos médios em DAP foram similares aos da testemunha não explorada (T7). De modo contrário, as espécies Pouteria bilocularis e Eschweilera grandiflora tiveram valores de IPA ${ }_{\text {DAP }}$ significativamente maiores nos tratamentos em que ocorreram intervenções na floresta (T1, T2, T3 e T6) do que na floresta não explorada (Tabela 3).

Eschweilera coriacea teve taxas de IPA ${ }_{\text {DAP }}$ superiores nos tratamentos $\mathrm{T} 2$ e $\mathrm{T} 6$ em relação à testemunha não explorada (T7). Por outro lado, os tratamentos com algum tipo de intervenção silvicultural (T1, T2, T3 e T6) mostraram não haver, ente si, diferenças significativas nas taxas 
TABELA 2: Mudanças no número de árvores com DAP igual ou maior que $35 \mathrm{~cm}$, em função da presença de cipós nas árvores selecionadas, em 300 ha de uma floresta natural de terra firme, município de Paragominas, Pará.

TABLE 2: Changes in the number of trees with DBH equal to or greater than $35 \mathrm{~cm}$, depending on the presence of climber on the trees selected in a 300 ha sample of natural terra firme forest, municipality of Paragominas, Pará.

\begin{tabular}{|c|c|c|c|c|c|c|c|c|c|}
\hline \multirow{2}{*}{ Tratamentos } & \multirow{2}{*}{$\begin{array}{c}\text { Categoria } \\
\text { de cipós }\end{array}$} & \multicolumn{2}{|c|}{2005} & \multicolumn{2}{|c|}{2006} & \multicolumn{2}{|c|}{2007} & \multicolumn{2}{|c|}{2009} \\
\hline & & $\mathrm{n}$ & $\%$ & $\mathrm{n}$ & $\%$ & $\mathrm{n}$ & $\%$ & $\mathrm{n}$ & $\%$ \\
\hline \multirow{7}{*}{$\mathrm{T} 1$} & Tipo I & 203 & 40,44 & 241 & 48,01 & 228 & 45,42 & 250 & 49,80 \\
\hline & Tipo II & 226 & 45,02 & 53 & 10,56 & 133 & 26,49 & 164 & 32,67 \\
\hline & Tipo III & 73 & 14,54 & 7 & 1,39 & 6 & 1,20 & 64 & 12,75 \\
\hline & Tipo IV & - & - & 1 & 0,20 & 17 & 3,39 & 1 & 0,20 \\
\hline & Tipo V & - & - & - & - & 16 & 3,19 & 1 & 0,20 \\
\hline & Tipo VI & - & - & 200 & 39,84 & 102 & 20,32 & 22 & 4,38 \\
\hline & Total & 502 & 100 & 502 & 100 & 502 & 100 & 502 & 100 \\
\hline \multirow{7}{*}{$\mathrm{T} 2$} & Tipo I & 155 & 37,62 & 196 & 47,57 & 242 & 58,74 & 207 & 50,24 \\
\hline & Tipo II & 202 & 49,03 & 31 & 7,52 & 56 & 13,59 & 162 & 39,32 \\
\hline & Tipo III & 55 & 13,35 & 2 & 0,49 & 7 & 1,70 & 26 & 6,31 \\
\hline & Tipo IV & - & - & 1 & 0,24 & 13 & 3,16 & 3 & 0,73 \\
\hline & Tipo V & - & - & - & - & 6 & 1,46 & 2 & 0,49 \\
\hline & Tipo VI & - & - & 182 & 44,17 & 88 & 21,36 & 12 & 2,91 \\
\hline & Total & 412 & 100 & 412 & 100 & 412 & 100 & 412 & 100 \\
\hline \multirow{7}{*}{$\mathrm{T} 3$} & Tipo I & 298 & 40,32 & 347 & 46,96 & 478 & 64,68 & 417 & 56,43 \\
\hline & Tipo II & 415 & 56,16 & 100 & 13,53 & 162 & 21,92 & 202 & 27,33 \\
\hline & Tipo III & 26 & 3,52 & 3 & 0,41 & 7 & 0,95 & 106 & 14,34 \\
\hline & Tipo IV & - & - & - & - & 20 & 2,71 & - & - \\
\hline & Tipo V & - & - & - & - & - & - & 2 & 0,27 \\
\hline & Tipo VI & - & - & 289 & 39,11 & 72 & 9,74 & 12 & 1,62 \\
\hline & Total & 739 & 100 & 739 & 100 & 739 & 100 & 739 & 100 \\
\hline
\end{tabular}

Em que: T1 = Exploração de Impacto Reduzido (EIR) + desbaste de liberação clássico e corte de cipós; T2 = EIR + desbaste de liberação modificado e corte de cipós; T3 = EIR + corte de cipós; Tipo I = nenhum cipó na árvore; Tipo II = cipós presentes, sem causar danos; Tipo III = cipós presentes, restringindo o crescimento; Tipo IV = cipós cortados, ainda vivos, porém, sem causar danos a árvore; Tipo $\mathrm{V}=$ cipós cortados, ainda vivos, restringindo o crescimento da árvore; Tipo VI = cipós cortados e mortos. n: número de indivíduos.

de crescimento. Silva et al. (2003) estudando o padrão de crescimento de árvores em floresta de terra firme na Amazônia Central detectaram para a espécie taxa de crescimento de $0,23 \mathrm{~cm}^{2} \mathrm{no}^{-1} \mathrm{e}$ coeficiente de variação (CV) de $78 \%$. Com base no valor de $\mathrm{CV}$ observado, os autores afirmaram haver indícios de que a espécie possui alguma elasticidade e deve responder favoravelmente aos tratamentos silviculturais. Esses indícios foram constatados também neste estudo, pois as variações nas taxas de IPA $_{\text {DAP }}$ verificadas para Eschweilera coriacea foram de aproximadamente $64 \%$ (T1) a $78 \%$ (T7).

Pouteria oblanceolata mostrou crescimento em DAP na floresta não explorada (T7) semelhante aos encontrados para os tratamentos com intervenção silvicultural (T1, T2, T3 e T6). Diferenças significativas foram verificadas apenas entre os tratamentos T3 e T6. Carvalho (1997) menciona que o padrão de crescimento em diâmetro de certas espécies pode ser semelhante em floresta sem perturbação e em floresta explorada, enquanto que de outras pode ser completamente diferente. Essa constatação foi observada em Pouteria oblanceolata mesmo depois de aplicadas as intervenções silviculturais pós-exploratórias.

Lecythis lurida encerrou taxas de crescimento em DAP similares nos tratamentos que envolveram algum tipo de intervenção silvicultural 
TABELA 3: Incremento periódico anual em DAP $\left(\mathrm{cm} \mathrm{ano}^{-1}\right)$ das oito espécies mais abundantes (DAP maior ou igual a $35 \mathrm{~cm}$ ), nos cinco tratamentos experimentais, no período de 2005 a 2009, em 500 ha de uma floresta natural de terra firme, município de Paragominas, Pará.

TABLE 3: Periodic annual DBH increment $\left(\mathrm{cm} \mathrm{year}^{-1}\right)$ of the eight most abundant species (DBH greater than or equal to $35 \mathrm{~cm}$ ) in the five experimental treatments, from 2005 to 2009 , in a 500 ha area of natural terra firme forest, municipality of Paragominas, Pará.

\begin{tabular}{|c|c|c|c|c|c|c|}
\hline Espécie & & $\mathrm{n}$ & $\mathrm{IPA}_{\mathrm{DAP}}$ & $\mathrm{CV}$ & $H$ & P-value \\
\hline \multirow{5}{*}{ Pouteria oblanceolata Pires } & T1 & 14 & $0,37 \mathrm{ab}$ & 43,22 & \multirow{5}{*}{11,35} & \multirow{5}{*}{0,0229} \\
\hline & $\mathrm{T} 2$ & 15 & $0,39 a b$ & 42,02 & & \\
\hline & T3 & 60 & $0,43 \mathrm{a}$ & 54,78 & & \\
\hline & T6 & 44 & $0,32 \mathrm{~b}$ & 57,14 & & \\
\hline & $\mathrm{T} 7$ & 56 & $0,31 \mathrm{ab}$ & 66,15 & & \\
\hline \multirow{5}{*}{ Protium subserratum (Engl.) Engl. } & $\mathrm{T} 1$ & 24 & 0,61 & 41,27 & \multirow{5}{*}{8,84} & \multirow{5}{*}{$\begin{array}{c}0,0651 \\
(\mathrm{~ns})\end{array}$} \\
\hline & $\mathrm{T} 2$ & 17 & 0,53 & 40,89 & & \\
\hline & $\mathrm{T} 3$ & 30 & 0,69 & 45,37 & & \\
\hline & T6 & 30 & 0,55 & 53,94 & & \\
\hline & $\mathrm{T} 7$ & 22 & 0,46 & 54,42 & & \\
\hline \multirow{5}{*}{ Pouteria bilocularis (H.K.A.Winkl.) Baehni } & $\mathrm{T} 1$ & 22 & $0,21 \mathrm{a}$ & 52,45 & \multirow{5}{*}{29,68} & \multirow{5}{*}{0,0001} \\
\hline & $\mathrm{T} 2$ & 29 & $0,23 \mathrm{a}$ & 62,32 & & \\
\hline & $\mathrm{T} 3$ & 29 & $0,20 \mathrm{a}$ & 57,40 & & \\
\hline & T6 & 36 & $0,25 \mathrm{a}$ & 51,72 & & \\
\hline & $\mathrm{T} 7$ & 44 & $0,10 \mathrm{~b}$ & 78,77 & & \\
\hline \multirow{5}{*}{ Lecythis lurida (Miers) S.A. Mori } & $\mathrm{T} 1$ & 24 & $0,37 \mathrm{ab}$ & 63,41 & \multirow{5}{*}{12,89} & \multirow{5}{*}{0,0118} \\
\hline & $\mathrm{T} 2$ & 18 & $0,37 \mathrm{ab}$ & 51,24 & & \\
\hline & T3 & 14 & $0,41 \mathrm{ab}$ & 62,20 & & \\
\hline & T6 & 22 & $0,42 \mathrm{a}$ & 59,83 & & \\
\hline & $\mathrm{T} 7$ & 18 & $0,18 \mathrm{~b}$ & 87,36 & & \\
\hline \multirow{5}{*}{ Eschweilera coriacea (DC.) S.A. Mori } & $\mathrm{T} 1$ & 34 & $0,35 \mathrm{ab}$ & 64,26 & \multirow{5}{*}{16,24} & \multirow{5}{*}{0,0027} \\
\hline & $\mathrm{T} 2$ & 27 & $0,54 \mathrm{a}$ & 66,65 & & \\
\hline & $\mathrm{T} 3$ & 29 & $0,39 a b$ & 74,24 & & \\
\hline & T6 & 38 & $0,43 \mathrm{a}$ & 65,85 & & \\
\hline & $\mathrm{T} 7$ & 62 & $0,28 \mathrm{~b}$ & 77,74 & & \\
\hline \multirow{5}{*}{ Eschweilera amazonica R. Knuth } & $\mathrm{T} 1$ & 53 & $0,19 \mathrm{ab}$ & 63,32 & \multirow{5}{*}{12,87} & \multirow{5}{*}{0,0119} \\
\hline & $\mathrm{T} 2$ & 32 & $0,18 \mathrm{ab}$ & 65,96 & & \\
\hline & $\mathrm{T} 3$ & 75 & $0,25 \mathrm{a}$ & 83,38 & & \\
\hline & T6 & 71 & $0,20 \mathrm{ab}$ & 82,84 & & \\
\hline & $\mathrm{T} 7$ & 26 & $0,12 \mathrm{~b}$ & 54,33 & & \\
\hline \multirow{5}{*}{ Eschweilera grandiflora (Aubl.) Sandwith } & $\mathrm{T} 1$ & 76 & $0,30 \mathrm{a}$ & 67,65 & \multirow{5}{*}{38,56} & \multirow{5}{*}{0,0001} \\
\hline & $\mathrm{T} 2$ & 60 & $0,44 \mathrm{~b}$ & 63,70 & & \\
\hline & $\mathrm{T} 3$ & 86 & $0,33 \mathrm{ab}$ & 60,97 & & \\
\hline & $\mathrm{T} 6$ & 83 & $0,30 \mathrm{ab}$ & 58,98 & & \\
\hline & $\mathrm{T} 7$ & 92 & $0,21 \mathrm{c}$ & 76,58 & & \\
\hline \multirow{5}{*}{ Laetia procera (Poepp.) Eichler } & $\mathrm{T} 1$ & 31 & 0,36 & 73,83 & \multirow{5}{*}{4,79} & \multirow{5}{*}{$\begin{array}{c}0,3085 \\
\text { (ns) }\end{array}$} \\
\hline & $\mathrm{T} 2$ & 17 & 0,31 & 89,93 & & \\
\hline & $\mathrm{T} 3$ & 17 & 0,43 & 71,49 & & \\
\hline & T6 & 30 & 0,43 & 60,67 & & \\
\hline & $\mathrm{T} 7$ & 21 & 0,31 & 69,65 & & \\
\hline
\end{tabular}

Em que: T1 = Exploração de Impacto Reduzido (EIR) + desbaste de liberação clássico e corte de cipós; T2 = EIR + desbaste de liberação modificado e corte de cipós; T3 = EIR + corte de cipós; T6 = apenas EIR; T7 = floresta não explorada (testemunha). $\mathrm{n}=$ número de indivíduos. $\mathrm{CV}=$ coeficiente de variação. $\mathrm{H}=$ valor da estatística de KruskalWallis. $P$-value $=$ indica o nível de significância do teste de Kruskal-Wallis para cada período de avaliação $(\alpha=0,05)$, $\mathrm{ns}=$ teste de Kruskal-Wallis não significativo. Tratamentos com letras diferentes, entre tratamentos experimentais, para cada espécie, são significativamente diferentes de acordo com o pós-teste de comparações múltiplas de Dunn $(\alpha=0,05)$. 
(T1, T2, T3 e T6). Contudo, crescimento em IPA ${ }_{\mathrm{DAP}}$ superior foi detectado para o tratamento T6 (apenas EIR) em relação ao T7 (testemunha não explorada). Estes resultados sugerem que, embora, a espécie seja enquadrada no grupo das espécies tolerantes à sombra (CARVALHO, 2000), suas taxas de crescimento são favorecidas pela abertura do dossel florestal, no caso, em especial, notadamente pela exploração de impacto reduzido. Jardim e Soares (2010) afirmam que embora algumas espécies arbóreas não dependam de radiação solar direta para se desenvolver, estas se beneficiam com a abertura do dossel através de clareiras, sejam naturais ou provenientes da exploração florestal.

Em termos gerais, a aplicação de tratamentos silviculturais após a exploração de impacto reduzido estimulou o crescimento de árvores quando comparado à floresta somente explorada ou à floresta não explorada. Dessa forma, ratificase a concepção de que a aplicação de tratamentos silviculturais a uma floresta na qual houve colheita de madeira é uma prática recomendável dentro de uma proposta de sistema silvicultural que seja aplicável às florestas naturais de terra firme da Amazônia brasileira. Não obstante, a definição de um sistema silvicultural pós-colheita "adequado" às florestas de terra firme da Amazônia brasileira deverá compatibilizar as boas práticas silviculturais aos menores custos operacionais.

\section{CONCLUSÃO}

Nos quatro anos de monitoramento da floresta, após a colheita de madeira e a aplicação dos tratamentos silviculturais, T2 (desbaste de liberação modificado e corte de cipós) e T3 (somente corte de cipós) tiveram as maiores taxas de crescimento em diâmetro. Contudo, o período de quatro anos não é suficiente para indicar o tratamento "mais adequado", com base no crescimento em diâmetro, em resposta à anelagem de árvores e corte de cipós.

Em nível de espécie os efeitos das intervenções silviculturais (colheita de madeira e/ou corte de cipós e/ou desbastes) nas taxas de IPA ${ }_{\mathrm{DAP}}$ mostraram-se não significativos para as espécies Protium subserratum e Laetia procera. As espécies Pouteria bilocularis e Eschweilera grandiflora tiveram valores de IPA ${ }_{\mathrm{DAP}}$ significativamente maiores nos tratamentos em que ocorreram intervenções na floresta (T1, T2, T3 e T6) do que na floresta não explorada.

\section{REFERÊNCIAS BIBLIOGRÁFICAS}

ALVINO, F. de O.; RAYOL, B. P.; SILVA, M. F. F. da. Avaliação de tratamentos silviculturais aplicados a espécies competidoras de Platonia insignis Mart. (Clusiaceae), em florestas secundárias na zona bragantina, Pará, Brasil. Revista de Ciências Agrárias, Belém, n. 45, p. 45-57, jan./jun. 2006. AMARAL, P. et al. Floresta para sempre: um manual para a produção de madeira na Amazônia. Belém: Imazon, 1998. $130 \mathrm{p}$.

AZEVEDO. C. P. de. et al. Efeito de diferentes níveis de exploração e de tratamentos silviculturais sobre a dinâmica da floresta remanescente. Floresta, Curitiba, v. 38, n. 2, p. 277-293, abr./jun. 2008.

CARVALHO, J. O. P. de. Dinâmica de florestas naturais e sua implicação para o manejo florestal. In: Tópicos em manejo florestal sustentável. Colombo, PR: Embrapa - CNPF. p. 43-55, 1997.

CARVALHO, J. O. P. de. Classificação em grupos ecológicos das espécies mais importantes em uma área de Floresta Nacional do Tapajós, Belterra, PA. Belém: Embrapa Amazônia Oriental, (Embrapa Amazônia Oriental. Comunicado Técnico, 41). 4 p. 2000.

CARVALHO, J. O. P. de; SILVA, J. N. M.; LOPES, J. do C. A. Growth rate of a terra firme rain forest in brazilian amazonia over an eight-year period in response to logging. Acta Amazônica, Manaus, v. 34, n. 2, p. 209-217. 2004.

COSTA, D. H. M.; SILVA, S. M. A. da; SILVA, J. N. M. Efetividade e custos do desbaste com aplicação de arboricida em floresta natural na região do Tapajós, Pará e Jari, Amapá. In: Silva, J. N. M.; Carvalho, J. O. P. de; Yared, J. A. C. (Ed.). A silvicultura na Amazônia Oriental: contribuições do projeto Embrapa-DFID. Belém: Embrapa Amazônia Oriental: DFID. 2001. p. 339352.

DE GRAAF, N. R. de. A silvicultural system for natural regeneration of tropical rain forest in Suriname. Wageningen: Agricultural University. 1986. 250 p.

DE GRAAF, N. R.; POELS, R. L. H.; VAN ROMPAEY, R. S. A. R. Effect of silvicultural treatment on growth and mortality of rainforest in Surinam over long periods. Forest Ecology and Management, EUA. v. 124, p. 123-135. 1999.

HUTCHINSON, I. D.; WADSWORTH, F. H. Efectos de la liberación en un bosque secundario de Costa Rica. Recursos Naturales y Ambiente. n. 46-47, p. 152-157. 2006. 
JARDIM, F. C. da S. et al. Dinâmica da vegetação arbórea com DAP maior ou igual a $5,0 \mathrm{~cm}$ : comparação entre grupos funcionais e ecofisiológicos na estação experimental de silvicultura tropical do INPA, Manaus-AM. Revista Árvore, Viçosa, v. 20, n. 3, p. 267-278. 1996.

JARDIM, F. C. da S.; SOARES, M. da S. Comportamento de Sterculia pruriens (Aubl.) Schum. em floresta tropical manejada em Moju-PA. Acta Amazônica, Manaus, v. 40, n. 3, p. 535-542. 2010.

MACIEL, M. de N. et al. Uso de imagens orbitais na estimativa de parâmetros estruturais de uma floresta primária no município de Paragominas, Estado do Pará. Revista de Ciências Agrárias, Belém, n. 52, p. 159-178, jul./dez. 2009.

OLIVEIRA, L. C. de et al. Efeito da exploração de madeira e tratamentos silviculturais na composição florística e diversidade de espécies em uma área de 136 ha na Floresta Nacional do Tapajós, Belterra, Pará. Scientia Forestalis, Piracicaba. n. 69, p. 62-76, dez. 2005.

OLIVEIRA, L. C. de et al. Eficiência de anelamento aplicado como tratamento silvicultural em florestas manejadas na Amazônia ocidental. Rio Branco. Comunicado Técnico $\mathrm{n}^{\circ} 172$. 2009. 9 p.

PEÑA-CLAROS, $M$. et al. Regeneration of commercial tree species following silvicultural treatments in a moist tropical forest. Forest Ecology and Management. v. 255, p. 1283-1293. 2008.

PÉREZ-SALICRUP, D. R. et al. Cost and efficiency of cutting lianas in a lowland liana forest of Bolivia.

Biotropica. v. 33, n. 2, p. 324-329. 2001.

RODRIGUES, T. E. et al. Caracterização e classificação dos solos do município de Paragominas, Estado do Pará. Belém: Embrapa Amazônia Oriental, (Embrapa Amazônia Oriental. Documentos, 162). 2003. 51 p.

SABOGAL, C. et al. Silvicultura na Amazônia brasileira: avaliação de experiências e recomendações para implementação e melhoria dos sistemas. 1. ed. Belém: Embrapa - CIFOR, 2006. $190 \mathrm{p}$.

SANDEL, M. P.; CARVALHO, J. O. P. de. Anelagem de árvores como tratamento silvicultural em florestas naturais da Amazônia brasileira. Revista de Ciências Agrárias, Belém, n. 33, p. 9-32. 2000.

SCHULZE, M.; GROGAN, J.; VIDAL, E. O manejo florestal como estratégia de conservação e desenvolvimento socioeconômico na Amazônia: quanto separa os sistemas de exploração madeireira atuais do conceito de manejo florestal sustentável? In: Nurit Bensusan, N.; Armstrong, G (eds.). O manejo da paisagem e a paisagem do manejo. Brasília: Instituto Internacional de Educação do Brasil, 2008. 161-213 p.

SILVA, J. N. M. et al. Growth and yield of a tropical rain forest in the Brazilian Amazon 13 years after logging. Forest Ecology and Management, Amsterdam. v. 71, p. 267-274. 1995.

SILVA, J. N. M. et al. Growth and yield studies in the Tapajós region, Central Brazilian Amazon. Commonwealth Forestry Review. v. 75, n. 4, p. 325-329. 1996.

SILVA, J. N. M.; CARVALHO, J. O. P. de; LOPES, J. do C. A. Um sistema silvicultural policíclico para produção sustentada de madeira na Amazônia brasileira. In: Simpósio Silvicultura na Amazônia Oriental: Contribuições do Projeto Embrapa/DFID. Documentos, 123. Belém: Embrapa-CPATU. 1999. p. $180-185$.

SILVA, J. N. M. et al. Crescimento, mortalidade e recrutamento em florestas de terra firme da Amazônia Oriental: observações nas regiões do Tapajós e Jarí. In: Silva, J. N. M.; Carvalho, J. O. P. de; Yared, J. A. G. (Ed.) A silvicultura na Amazônia Oriental: contribuições do projeto Embrapa/DFID. Belém: Embrapa Amazônia Oriental/DFID. 2001. p. 291-308.

SILVA, J. N. M. Manejo Florestal. 3. ed. Brasília: Embrapa Informação Tecnológica, v.1. 2001. 49 p. SILVA, R. P. da et al. Uso de banda dendrométrica na definição de padrões de crescimento individual em diâmetro de árvores da bacia do rio cuieiras. Acta Amazônica, Manaus, v. 33, n. 1, p. 67-84. 2003.

SILVA, J. N. M. et al. Diretrizes para instalação e medição de parcelas permanentes em florestas naturais da Amazônia brasileira. 1. ed. Belém: Embrapa, 2005. 68 p.

VELOSO, H. P.; RANGEL FILHO, A. L. R.; LIMA, J. C. A. 1991. Classificação da vegetação brasileira adaptada a um sistema universal. IBGE, Departamento de recursos naturais e estudos ambientais, Rio de Janeiro. 124 p.

VILLEGAS, Z. et al. Silvicultural treatments enhance growth rates of future crop trees in a tropical dry forest. Forest Ecology and Management. v. 258, p. 971-977. 2009.

WADSWORTH, F. H. Producción forestal para América Tropical. Washington: USDA, 2000. $602 \mathrm{p}$. 
WADSWORTH, F. H.; ZWEEDE, J. C. Liberation: acceptable production of tropical forest timber. Forest Ecology and Management, Amsterdam. v. 233, n. 1, p. 45-51. 2006. WATRIN, O. dos S.; ROCHA, A. M. A. Pesquisa, 124). 1992. 40 p.
Levantamento da vegetação natural e do uso da terra no município de Paragominas (PA) utilizando imagens TM/LANDSAT. Belém: Embrapa-CPATU. (Embrapa - CPATU. Boletim de 